Supporting information for

\title{
Polymeric Micelles with Uniform Surface Properties and Tunable Size and Charge: Positive Charges Improve Tumor Accumulation
}

\author{
Tong Shen ${ }^{\mathrm{a}}$, Shuli Guan ${ }^{\mathrm{a}}$ Zhihua Gan ${ }^{\mathrm{a}}$, Guan Zhang ${ }^{\mathrm{b} *}$ and Qingsong Yu $\mathrm{a}^{\mathrm{a}^{*}}$
}

${ }^{\mathrm{a}}$ The State Key laboratory of Organic-inorganic Composites, Beijing Laboratory of

Biomedical Materials, College of Life Science and Technology, Beijing University of Chemical Technology, Beijing 100029, China

${ }^{b}$ Department of Urology, China-Japan Friendship Hospital, Beijing 100029, China

Figure S1. ${ }^{1} \mathrm{H}$ NMR spectra of diblock and triblock copolymers.

Figure S2. The alteration of size (left) and zeta potential (right) of micelles prepared with (A) THF, (B) DMSO and (C) DMF after TFA was added into micelle solution.

Figure S3. The size distribution of polymeric micelles during deprotection process.

Figure S4. Temperature related light absorbance at $500 \mathrm{~nm}$ of diblock polymeric micelles before and after treated with TFA or HAc.

Figure S5. ${ }^{1} \mathrm{H}$ NMR spectra of polymeric micelles recorded in deuterium oxide after the addition of different amount of deuterated TFA.

Figure S6. The stability assay of triblock copolymer micelles in $50 \%$ Fetal Bovine Serum.

Figure S7. The in vivo biodistribution of triblock polymeric micelles with different surface charges in three paralleled 4T1 tumor bearing mice.

Figure S8. The mean fluorescent intensities of triblock polymeric micelles with different surface charges in isolated organs.

Figure S9. The tumor to normal organ (tumor to liver (T/L), tumor to kidney $(\mathrm{T} / \mathrm{K})$, tumor to spleen $(\mathrm{T} / \mathrm{S})$ ) fluorescent intensity ratios of triblock polymeric micelles with different surface charges.

Figure S10. The in vivo biodistribution of diblock polymeric micelles with different surface charges in $4 \mathrm{~T} 1$ tumor bearing mice.

\footnotetext{
*To whom correspondence should be addressed. E-mail: gzhang40@hotmail.com (Guan Zhang); 


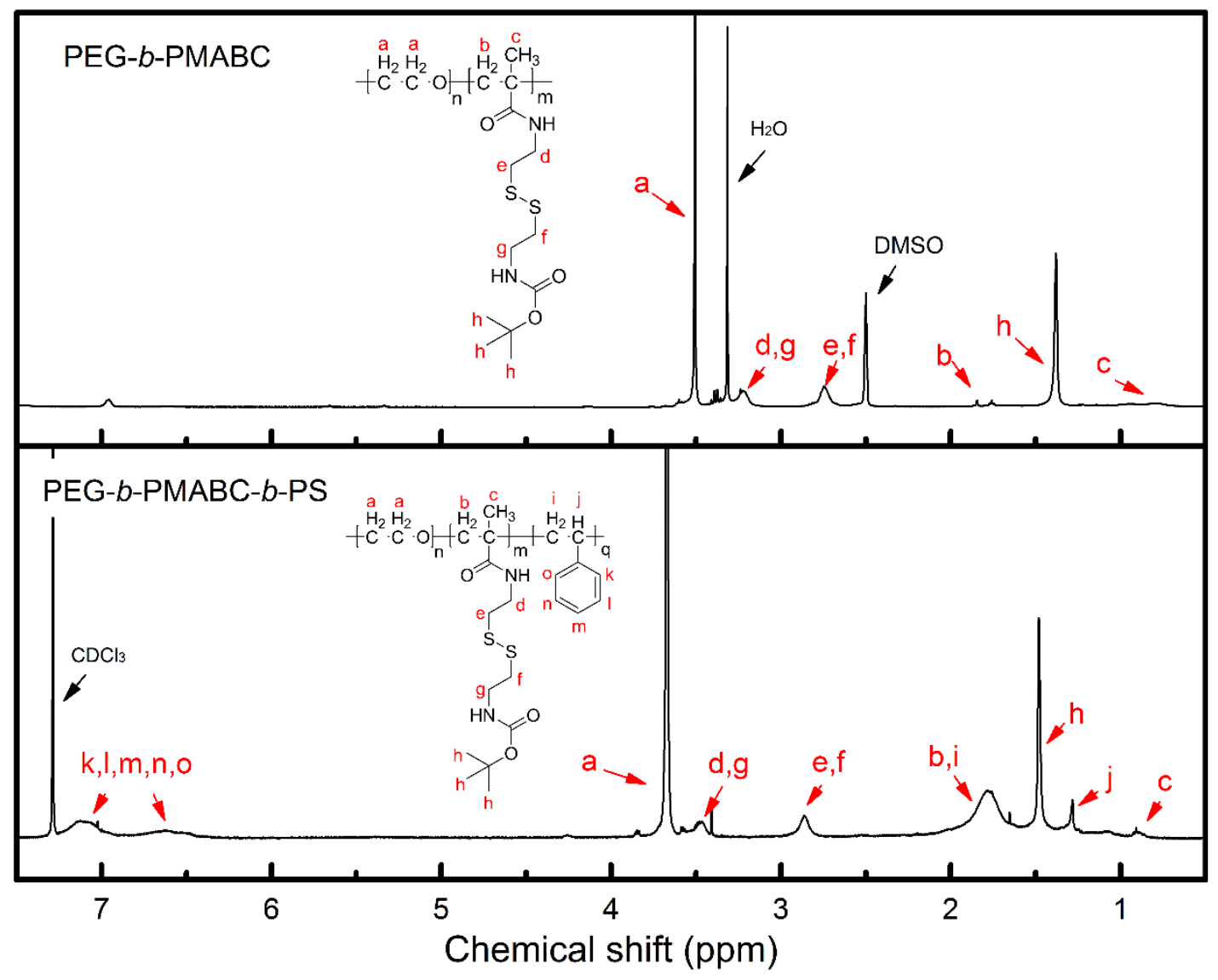

Figure S1. ${ }^{1}$ H NMR spectra of diblock and triblock copolymers. 

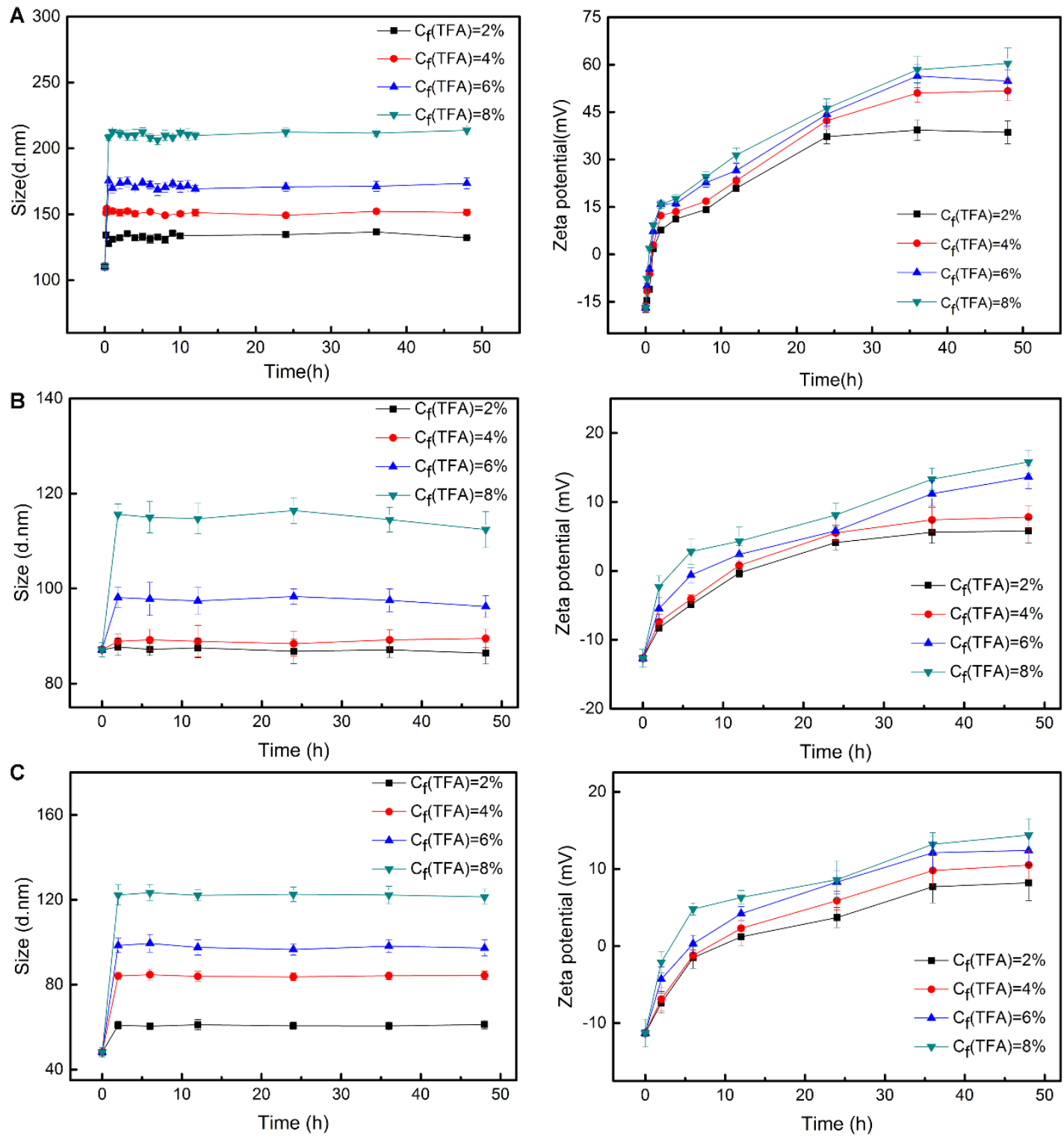

Figure S2. The alteration of size (left) and zeta potential (right) of micelles prepared with (A) THF, (B) DMSO and (C) DMF after TFA was added into micelle solution. 

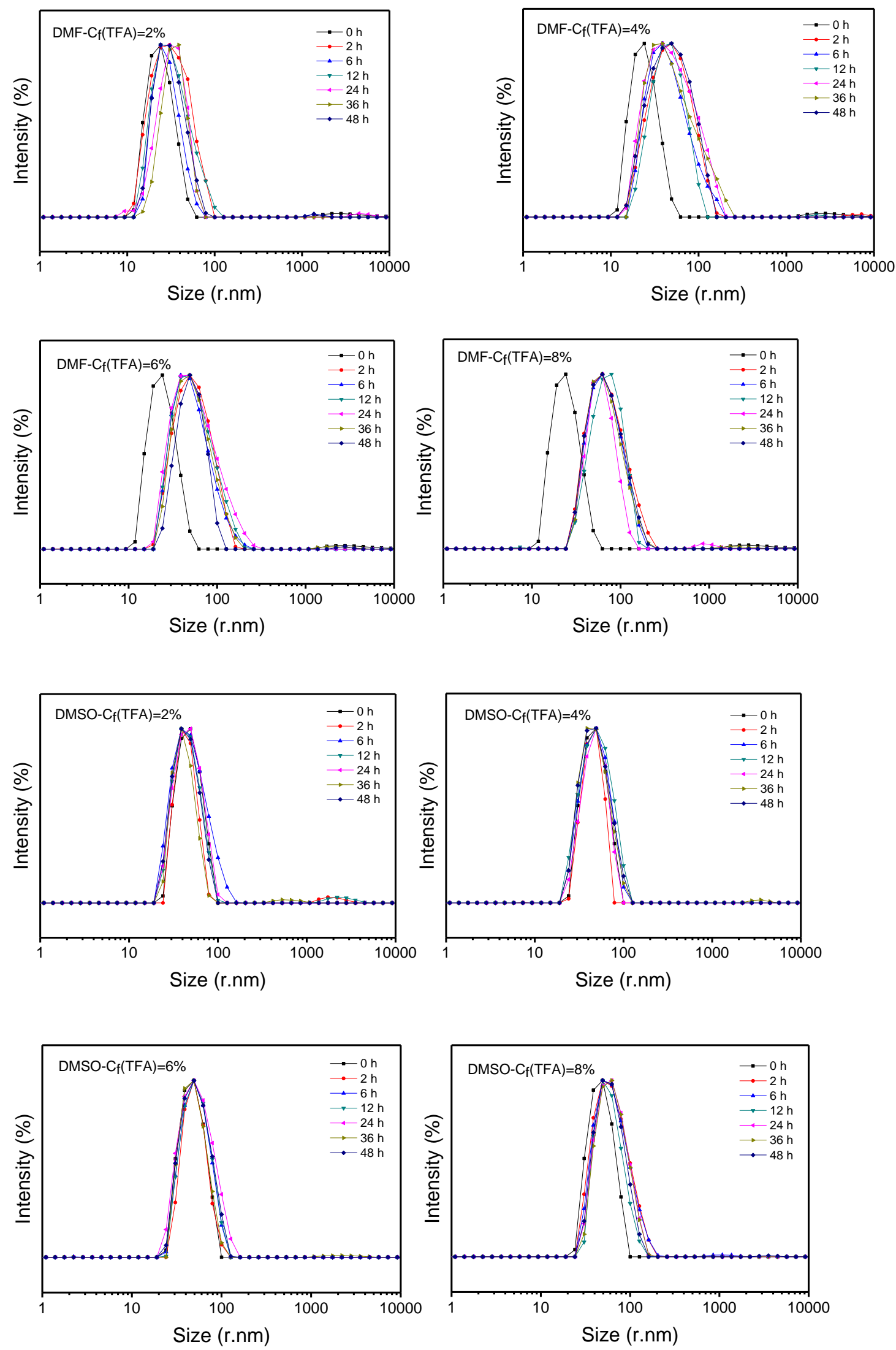

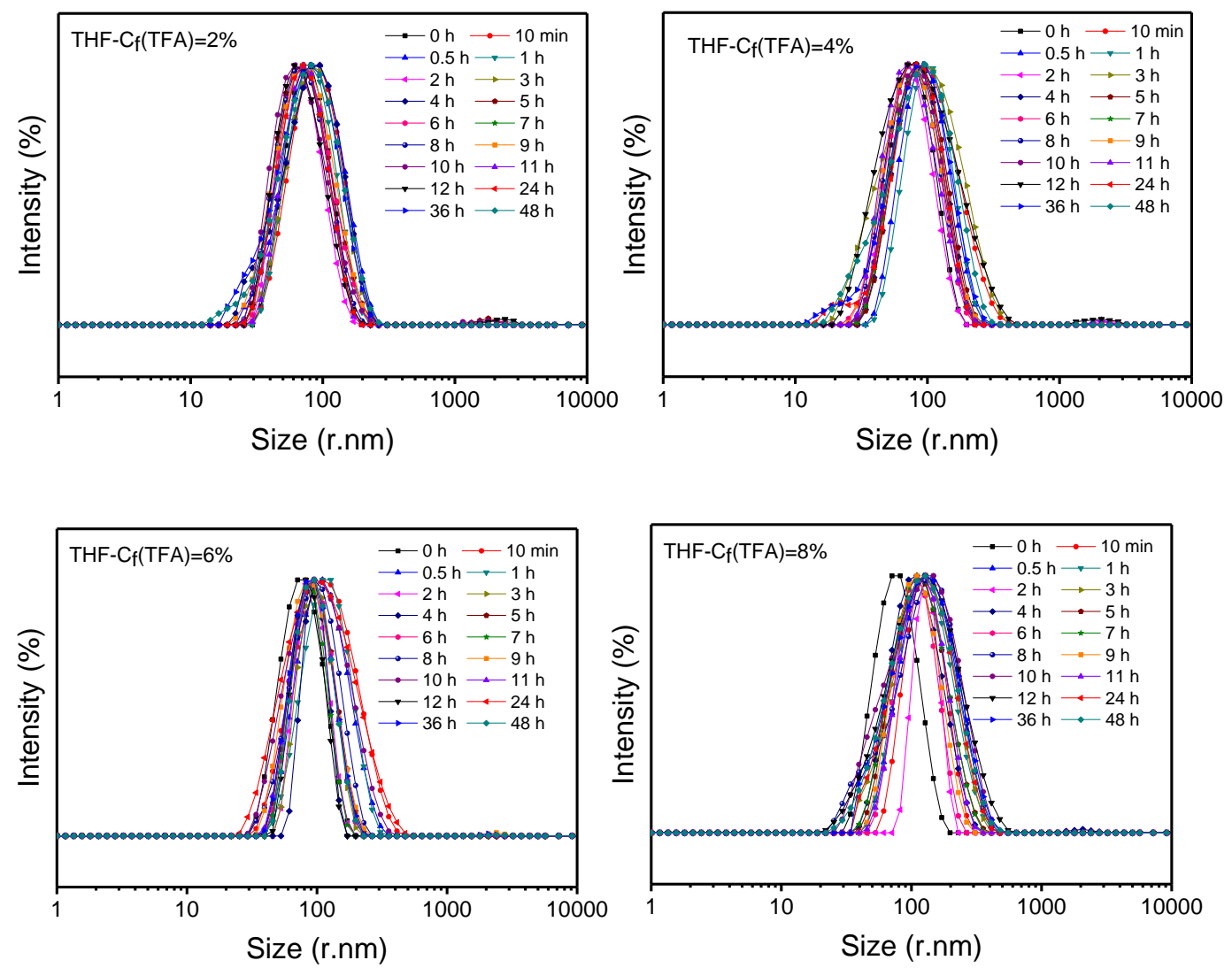

Figure S3. The size distribution of polymeric micelles during deprotection process.

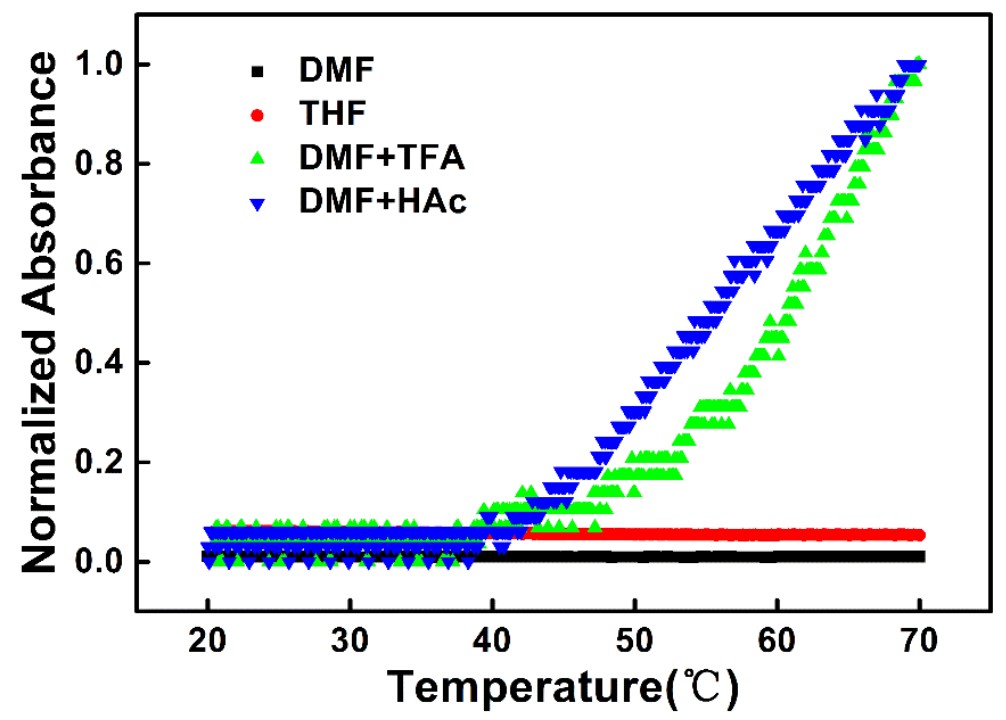

Figure S4. Temperature related light absorbance at $500 \mathrm{~nm}$ of diblock polymeric micelles before and after treated with TFA or HAc. 


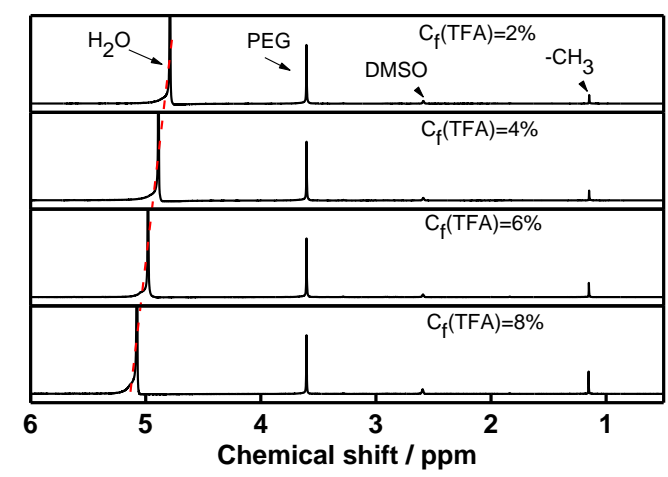

Figure S5. ${ }^{1} \mathrm{H}$ NMR spectra of polymeric micelles recorded in deuterium oxide after the addition of different amount of deuterated TFA.
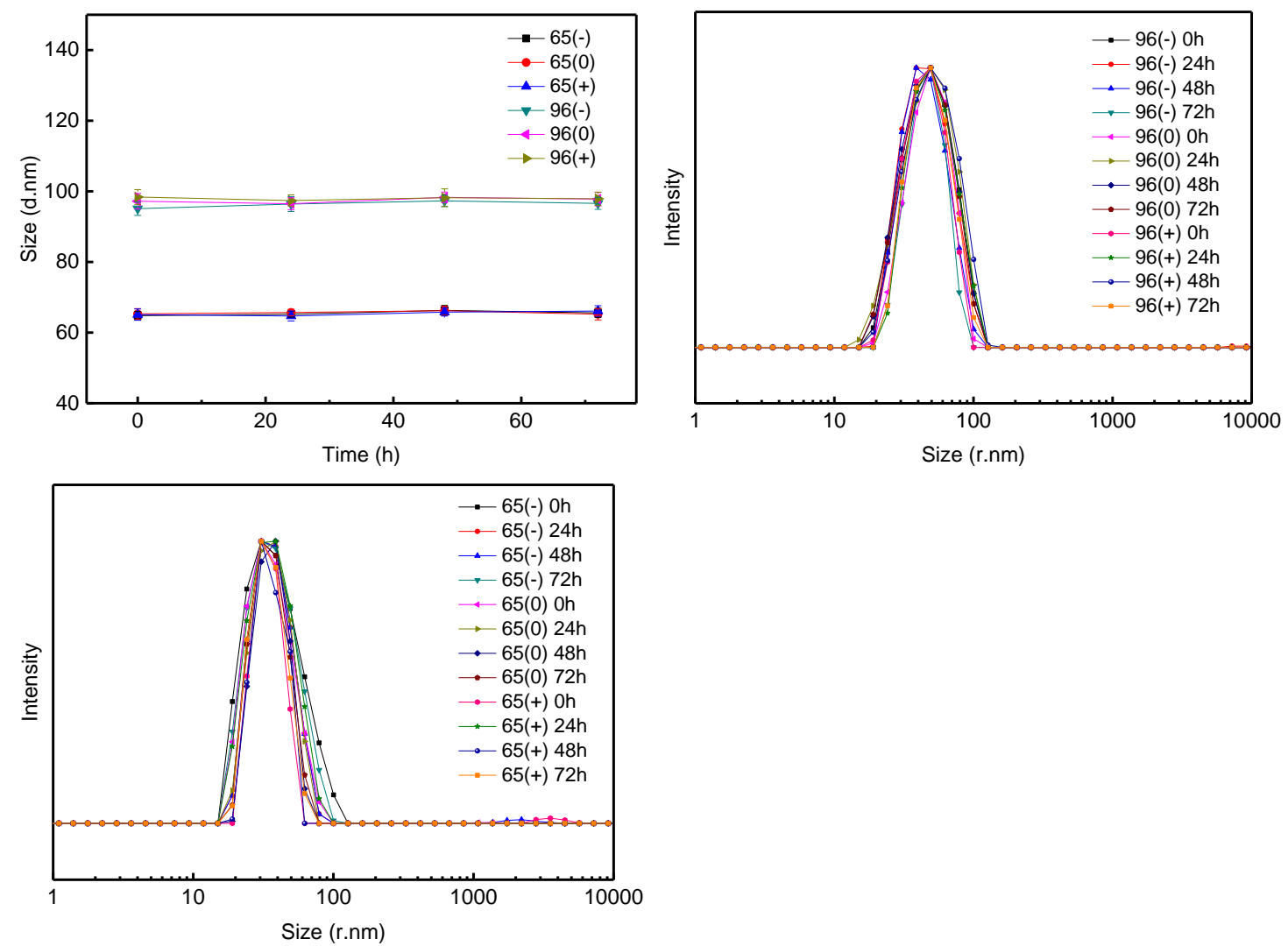

Figure S6. The stability assay of triblock copolymer micelles in 50\% Fetal Bovine Serum. 
$65 \mathrm{~nm}(-)$

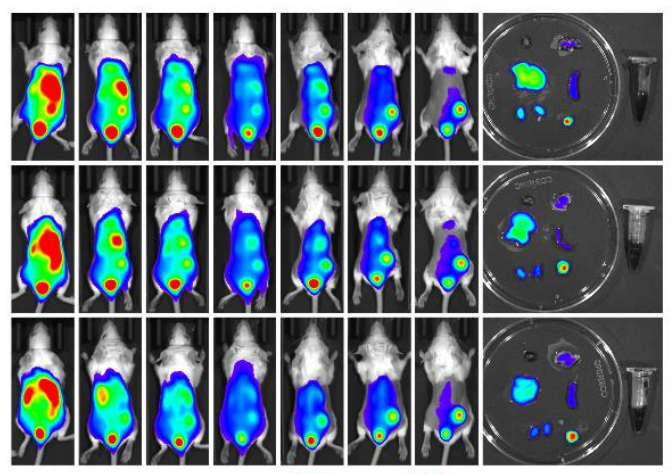

$65 \mathrm{~nm}(0)$

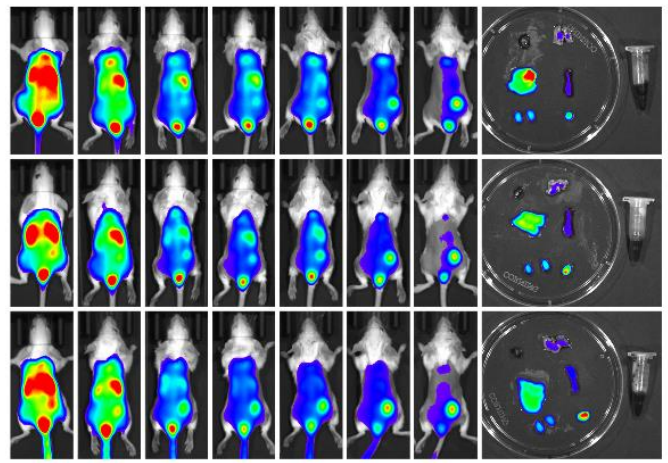

$65 \mathrm{~nm}(+)$
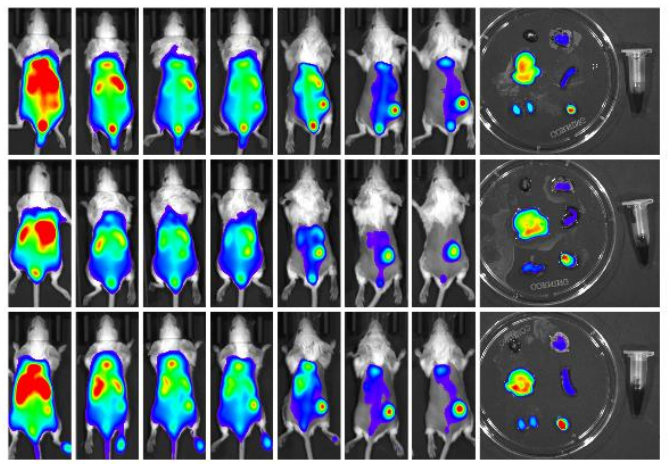

$96 \mathrm{~nm}(-)$

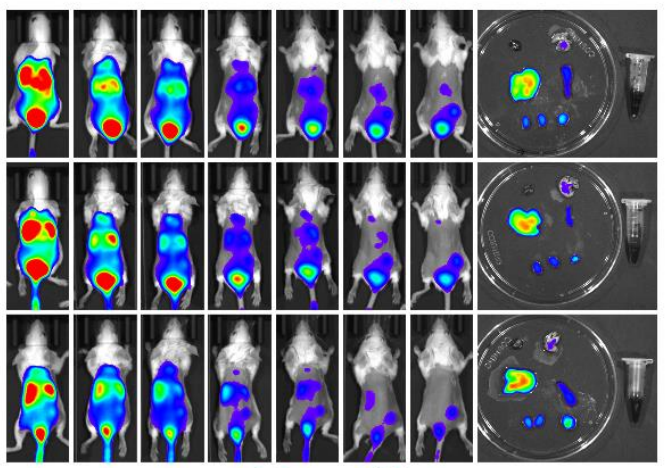

$96 \mathrm{~nm}(0)$

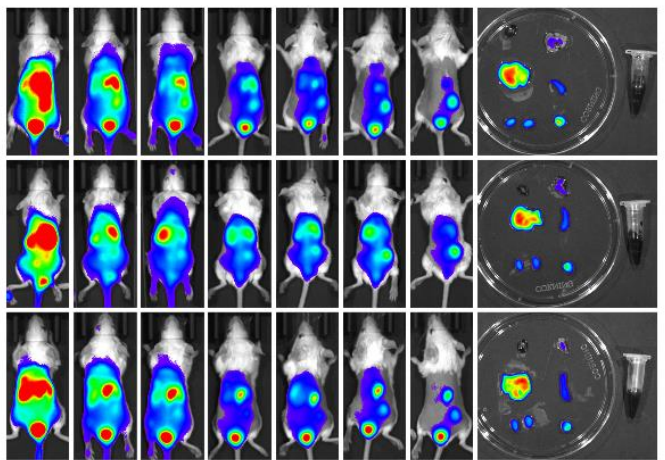

$96 \mathrm{~nm}(+)$
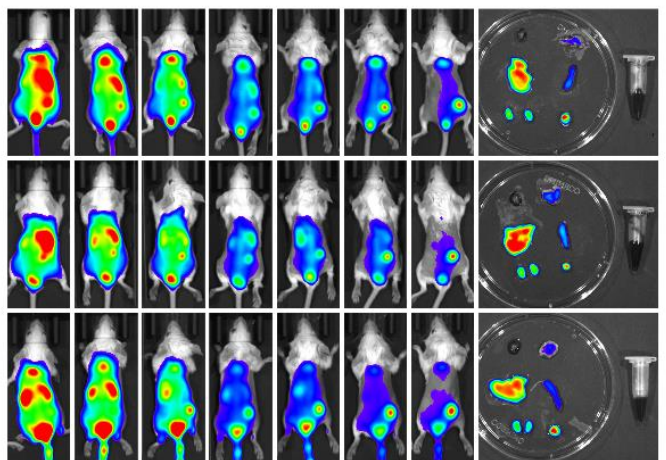

Figure S7. The in vivo biodistribution of triblock polymeric micelles with different surface charges in three paralleled 4T1 tumor bearing mice. 


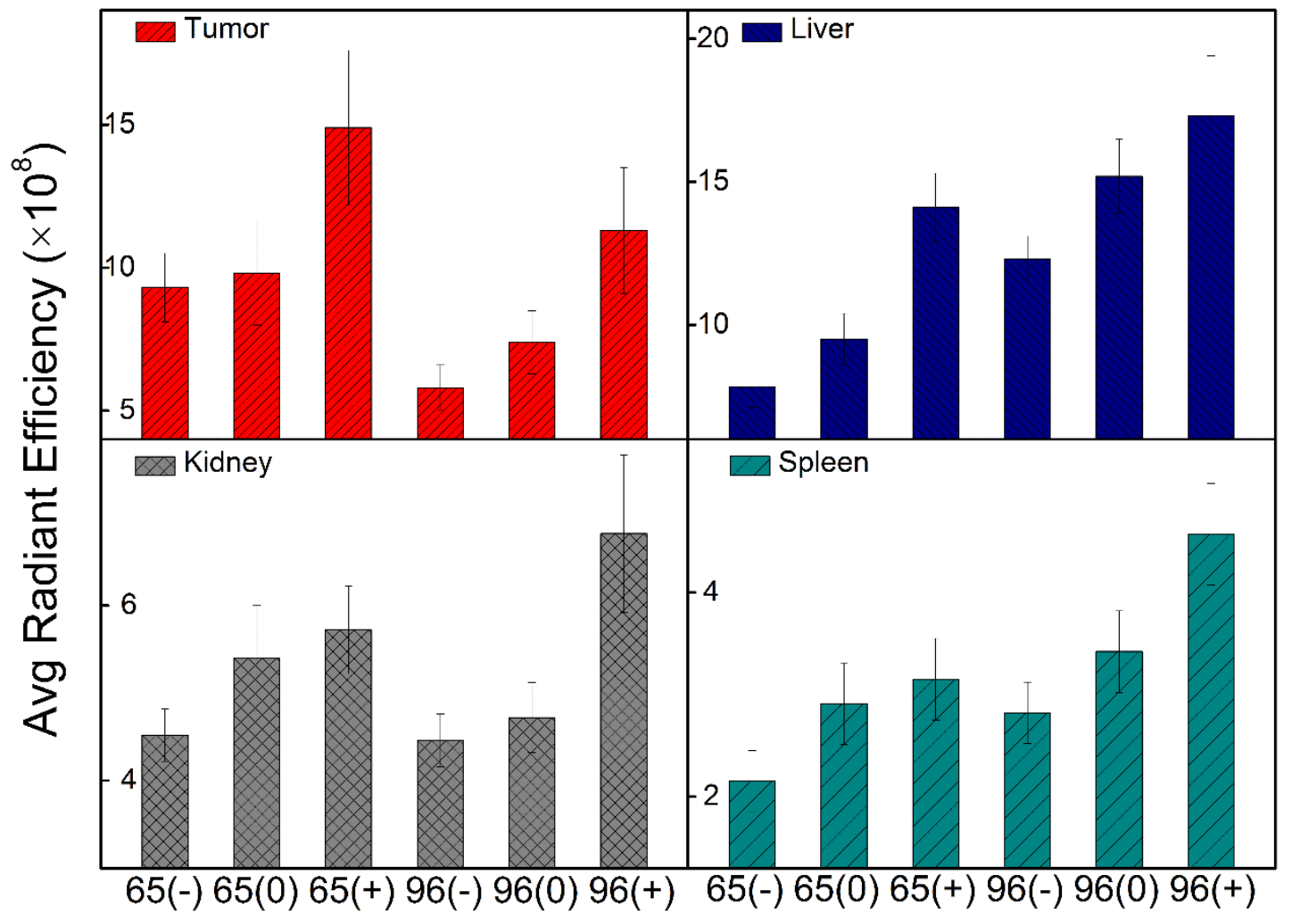

Figure S8. The mean fluorescent intensities of triblock polymeric micelles with different surface charge in isolated organs. 


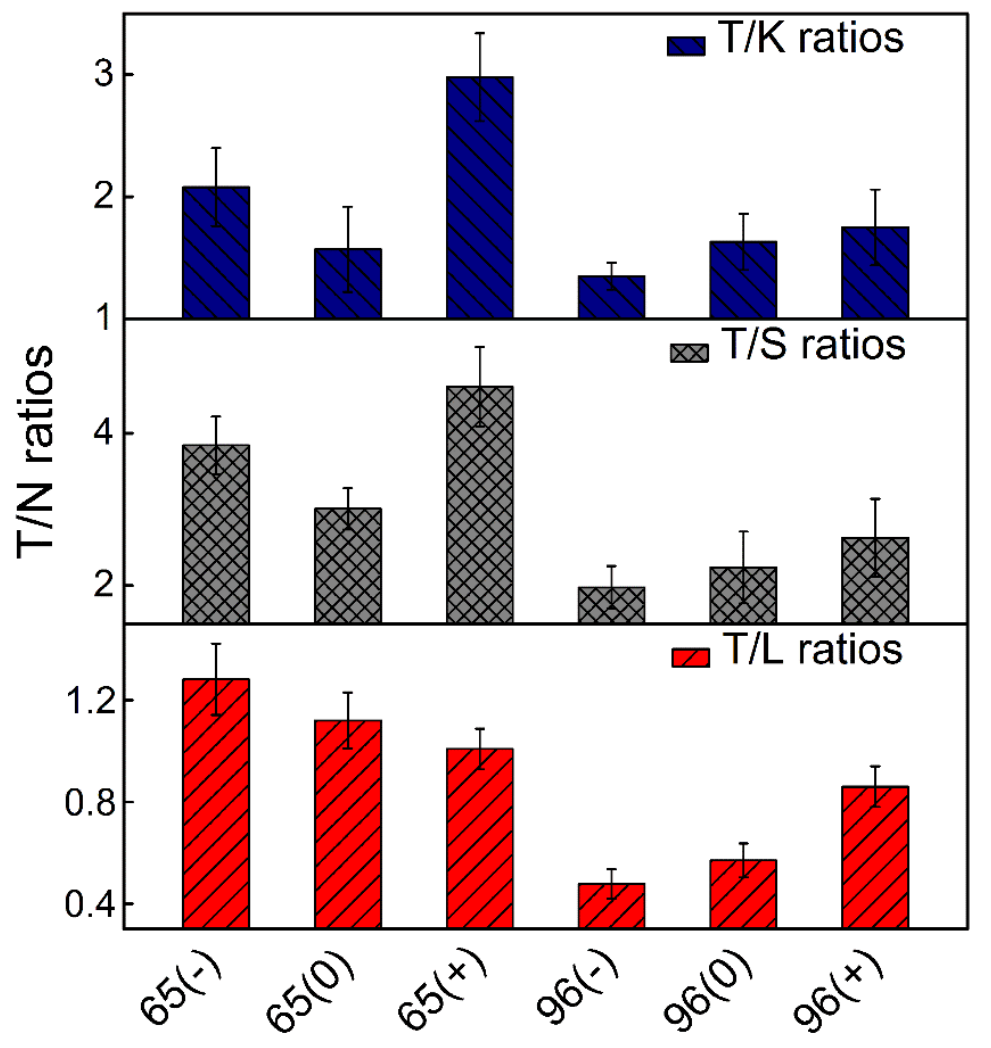

Figure S9. The tumor to normal organ (tumor to liver $(\mathrm{T} / \mathrm{L})$, tumor to kidney $(\mathrm{T} / \mathrm{K})$, tumor to spleen $(\mathrm{T} / \mathrm{S}))$ fluorescent intensity ratios of triblock polymeric micelles with different surface charge. 


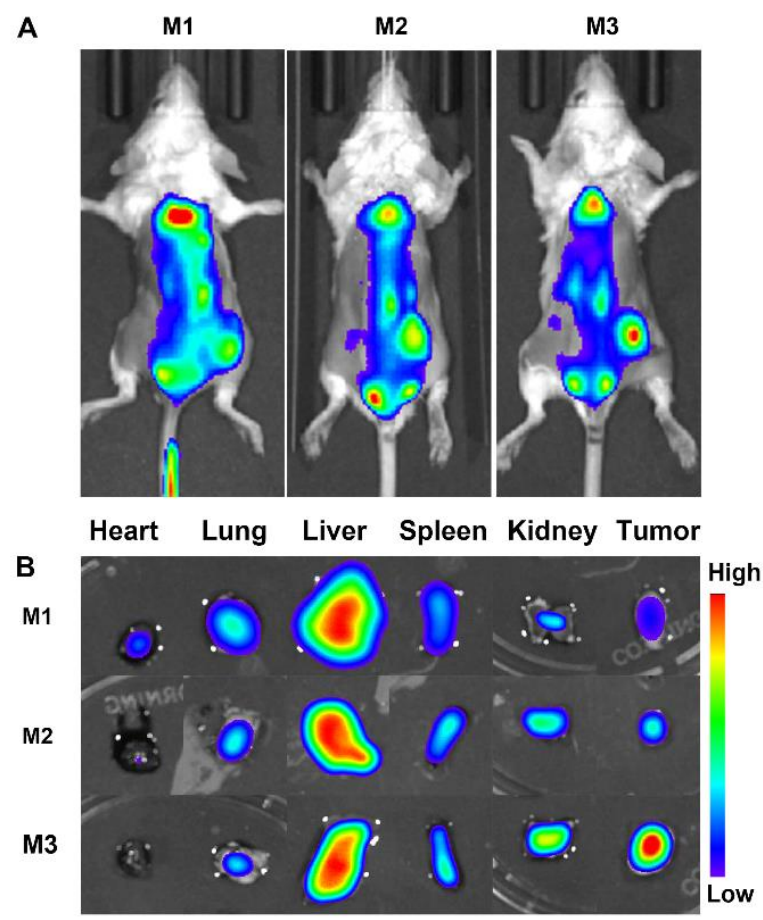

Figure S10. The in vivo biodistribution of diblock polymeric micelles with different surface charge in $4 \mathrm{~T} 1$ tumor bearing mice. 